\title{
LOWNESS FOR EFFECTIVE HAUSDORFF DIMENSION
}

\author{
STEFFEN LEMPP, JOSEPH S. MILLER, KENG MENG NG, DANIEL D. TURETSKY,
} AND REBECCA WEBER

\begin{abstract}
We examine the sequences $A$ that are low for dimension, i.e., those for which the effective (Hausdorff) dimension relative to $A$ is the same as the unrelativized effective dimension. Lowness for dimension is a weakening of lowness for randomness, a central notion in effective randomness. By considering analogues of characterizations of lowness for randomness, we show that lowness for dimension can be characterized in several ways. It is equivalent to lowishness for randomness, namely, that every Martin-Löf random sequence has effective dimension 1 relative to $A$, and lowishness for $K$, namely, that the limit of $K^{A}(n) / K(n)$ is 1 .

We show that there is a perfect $\Pi_{1}^{0}$-class of low for dimension sequences. Since there are only countably many low for random sequences, many more sequences are low for dimension. Finally, we prove that every low for dimension is jump-traceable in order $n^{\varepsilon}$, for any $\varepsilon>0$.
\end{abstract}

\section{INTRODUCTION}

The effective (Hausdorff) dimension of an infinite sequence $A \in 2^{\omega}$ is

$$
\operatorname{dim}(A)=\liminf _{n \rightarrow \infty} \frac{K(A\lceil n)}{n} .
$$

This notion was introduced by Lutz [15], who effectivized a martingale characterization of Hausdorff dimension and defined $\operatorname{dim}(A)$ to be the effective Hausdorff dimension of $\{A\}$. The characterization above, which reveals effective dimension to be a natural measure of the information density of infinite sequences, was given by Mayordomo [16] (see also Ryabko [20]).

We say that $A \in 2^{\omega}$ is low for (effective Hausdorff) dimension if

$$
\left(\forall X \in 2^{\omega}\right)\left[\operatorname{dim}^{A}(X) \geq \operatorname{dim}(X)\right] .
$$

In other words, $A$ is too weak as an oracle to change the effective Hausdorff dimension of any sequence. It is clear that $\operatorname{dim}^{A}(X) \leq \operatorname{dim}(X)$, for any $A \in 2^{\omega}$, so if $A$ is low for dimension we have $\operatorname{dim}^{A}(X)=\operatorname{dim}(X)$ for all $X$.

This paper initiates the study of lowness for dimension. Our main result gives several characterizations of the notion. These can be seen as weakenings of wellknown characterizations of lowness for randomness. This analogy helped direct our research and will be discussed in detail below.

Date: December 11, 2014.

2010 Mathematics Subject Classification. Primary 03D32; Secondary 68Q30, 28A78.

Lempp's research was partly supported by Grant \# 13407 by the John Templeton Foundation entitled "Exploring the Infinite by Finitary Means" as well as AMS-Simons Foundation Collaboration Grant 209087. Miller was supported by the National Science Foundation under grants DMS-0945187. Miller was also supported by NSF grant DMS-0946325 and Weber by NSF grant DMS-0652326, both part of a Focused Research Group in Algorithmic Randomness. 
We need a few more definitions before we can state the main theorem. If $\sigma \in 2^{<\omega}$, then we write $[\sigma]$ for the clopen set $\left\{X \in 2^{\omega}: \sigma \prec X\right\}$. We extend this to a set of strings $W \subseteq 2^{<\omega}$ by letting $[W]$ be the open set $\bigcup_{\sigma \in W}[\sigma]$. Finally, if $W \subseteq 2^{<\omega}$ and $s \in[0,1]$, then the $s$-dimensional weight of $W$ is $\operatorname{wt}_{s}(W)=\sum_{\sigma \in W} 2^{-s|\sigma|}$.

Main Theorem. The following are equivalent for $A \in 2^{\omega}$ :

(1) $A$ is lowish for (Martin-Löf) randomness, i.e., if $X \in 2^{\omega}$ is Martin-Löf random, then $\operatorname{dim}^{A}(X)=1$.

(2) There is an $s \in(0,1]$ such that $\left(\forall X \in 2^{\omega}\right)\left[\operatorname{dim}(X)=s \Rightarrow \operatorname{dim}^{A}(X)=s\right]$.

(3) $A$ is low for dimension.

(4) $A$ has the $\Sigma_{1}^{0}$-covering property, i.e., if $W \subseteq 2^{<\omega}$ is $A$-c.e., $0 \leq s<t \leq 1$, and $\mathrm{wt}_{s}(W)<1$, then there is a c.e. set $V$ with $\mathrm{wt}_{t}(V)<1$ such that $[W] \subseteq[V]$.

(5) A has the c.e. covering property, i.e., if $W \subseteq 2^{<\omega}$ is $A$-c.e., $0 \leq s<t \leq 1$, and $\mathrm{wt}_{s}(W)<1$, then there is a c.e. set $V \supseteq W$ with $\mathrm{wt}_{t}(V)<\infty$.

(6) $\liminf _{\sigma \rightarrow \infty} \frac{K^{A}(\sigma)-K(\sigma)}{|\sigma|} \geq 0$.

(7) $A$ is lowish for $K$, i.e., $\liminf _{\sigma \rightarrow \infty} \frac{K^{A}(\sigma)}{K(\sigma)} \geq 1$.

Some of these properties are easily shown to be equivalent to superficially stronger forms. It is clear that $\limsup _{\sigma \rightarrow \infty} K^{A}(\sigma) / K(\sigma) \leq 1$, so $(7)$ is equivalent to

$$
\left(7^{\prime}\right) \lim _{\sigma \rightarrow \infty} \frac{K^{A}(\sigma)}{K(\sigma)}=1 .
$$

In the same way, (6) is equivalent to

$$
\left(6^{\prime}\right) \lim _{\sigma \rightarrow \infty} \frac{K^{A}(\sigma)-K(\sigma)}{|\sigma|}=0 .
$$

Finally, in (5) we can discard finitely many of the strings in $V \backslash W$ to ensure that $\mathrm{wt}_{t}(V)<1$.

Remark 1.1. We use prefix-free complexity, $K$, throughout this paper, but the main theorem would be unchanged if we had used plain complexity, $C$. To see this, note that $\lim _{\sigma \rightarrow \infty} C(\sigma) / K(\sigma)=1$, and of course this fact relativizes. So as is wellknown, we could have used $C$ in the definition of effective dimension. Furthermore, properties (6) and (7) are equivalent to the corresponding properties stated in terms of plain complexity.

Analogy with lowness for randomness. Recall that a sequence $A \in 2^{\omega}$ is $K$-trivial if $K\left(A\lceil n) \leq^{+} K(n),{ }^{1}\right.$ in other words, if its initial segment prefix-free complexity is as low as possible. The class of $K$-trivial sequences has been a rich area of study in recent years, and many diverse characterizations have been found. See Nies [19] or Downey and Hirschfeldt [6] for a thorough introduction to $K$ triviality. The characterizations of lowness for dimension in the main theorem are analogous to some of the characterizations of $K$-triviality (though not $K$-triviality itself). In particular:

Theorem 1.2. The following are equivalent for $A \in 2^{\omega}$ :

(a) A is K-trivial.

\footnotetext{
${ }^{1}$ We use $f(n) \leq+g(n)$ to mean $(\exists c)(\forall n)[f(n) \leq g(n)+c]$.
} 
(b) A is low for (Martin-Löf) randomness, i.e., every Martin-Löf random sequence is Martin-Löf random relative to $A$.

(c) $A$ is low for $K$, i.e., $K(\sigma) \leq^{+} K^{A}(\sigma)$.

(d) Every $\Sigma_{1}^{0}[A]$-class of measure less than one is contained in a $\Sigma_{1}^{0}$-class of measure less than one.

It is easy to see that (c) implies (a) and (b). The equivalence of (a)-(c) was proved in Nies [17], where the proof that every $K$-trivial is low for $K$ is attributed to Hirschfeldt and Nies. Kjos-Hanssen [11] proved that (d) is equivalent to the others.

There is an obvious similarity between the properties in the main theorem and Theorem 1.2. Property (1) is a direct weakening of (b), though (2) and (3) have a similar feel. Properties (6) and (7) are both natural weakenings of (c), while property (4) is a natural analogue of (d). Property (5) is also analogous to a known characterization of $K$-triviality; see Simpson [21, Lemma 5.11] for a strong version. $^{2}$

It is clear that lowness for randomness implies lowness for dimension. What about the converse? Hirschfeldt and Weber [10] constructed a sequence $A \in 2^{\omega}$ that has finite self-information (see Section 7) but is not low for randomness. They observed that their sequence is also low for dimension, so lowness for dimension does not imply lowness for randomness. We can also see this as a corollary to Theorem 5.1, where we prove that there are continuum many sequences that are low for dimension (see also Herbert [9]). Chaitin [4] proved that every $K$-trivial is $\Delta_{2}^{0}$, so there are only countably many low for random sequences.

Structure of the paper. The next three sections are dedicated to the proof of the main theorem. The equivalence of the seven properties will be shown as follows:
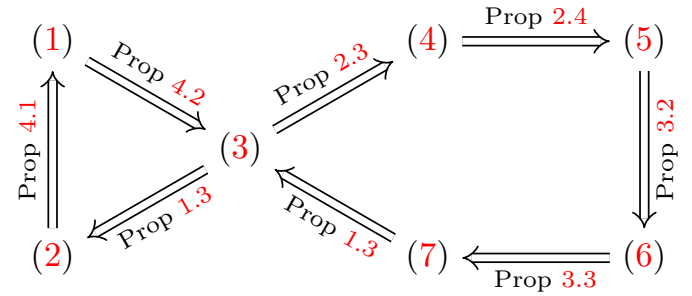

There are several trivial implications between the various properties, two of which appear in the diagram.

\section{Proposition 1.3.}

- $(3) \Rightarrow(2)$.

- $(7) \Rightarrow(3)$.

Proof. If $A$ is low for dimension, then for every $X \in 2^{\omega}$ and $s \in[0,1]$, we have $\operatorname{dim}(X)=s$ implies $\operatorname{dim}^{A}(X)=s$. So it is clear that (3) implies (2).

\footnotetext{
${ }^{2}$ In light of Remark 1.1, we should note that $C$-trivial and low for $C$ are not equivalent to $K$ trivial and low for $K$. It is easy to see that every low for $C$ sequence is $C$-trivial, and Chaitin [3] proved that such sequences are computable.
} 
Now assume that $A$ is lowish for $K$, i.e., satisfies (7). Then for any $X \in 2^{\omega}$,

$$
\begin{aligned}
\operatorname{dim}^{A}(X)=\liminf _{n \rightarrow \infty} \frac{K^{A}(X\lceil n)}{n} & =\liminf _{n \rightarrow \infty} \frac{K(X\lceil n)}{n} \frac{K^{A}(X\lceil n)}{K(X\lceil n)} \\
& \geq \liminf _{n \rightarrow \infty} \frac{K(X\lceil n)}{n}=\operatorname{dim}(X) .
\end{aligned}
$$

So $A$ is low for dimension, i.e., (3) holds.

In Section 5, we produce a perfect $\Pi_{1}^{0}$-class of low for dimension sequences, proving that there are continuum many. In Section 6, we investigate the degrees of sequences that are low for dimension. The main result in that section is that if $A \in 2^{\omega}$ is low for dimension and $\varepsilon>0$, then $A$ is jump-traceable with bound $n^{\varepsilon}$. No slower-growing bound is known to permit continuum many jump-traceable sequences. The final section presents a few open questions related to our work.

We assume that the reader is familiar with elementary computability theory and algorithmic randomness. Downey and Hirschfeldt [6] and Nies [19] are excellent references for these topics, although Nies does not cover effective dimension. One tool that we use repeatedly is the bounded request set. Let $W$ be a c.e. set of requests of the form $\langle\sigma, d\rangle$ where $\sigma \in 2^{<\omega}$ and $d \in \omega$. We call $W$ a bounded request set if $\sum_{\langle\sigma, d\rangle \in W} 2^{-d}<\infty$. $^{3}$ If $W$ is a bounded request set, then $(\forall\langle\sigma, d\rangle \in$ $W)\left[K(\sigma) \leq^{+} d\right]$. This follows from the Kraft-Chaitin Theorem.

\section{The COVERING PROPERTIES}

In this section, we show that if $A \in 2^{\omega}$ is low for dimension then it satisfies both covering properties. Although the statements of the covering properties are very similar, they are on two sides of a divide between properties involving infinite sequences and properties involving finite strings. This is what separates (1)-(4) from (5)-(7). Bridging this gap is the most delicate part of proving the main theorem.

Let $s \in[0,1]$ and $U \subseteq 2^{<\omega}$. The prefix-free s-weight of $U$ is $\operatorname{pfwt}_{s}(U)=$ $\sup \left\{\operatorname{wt}_{s}(V): V \subseteq U\right.$ is prefix-free $\}$. We say that $U$ is $s$-closed if for every $\tau \in 2^{<\omega}$, if $\operatorname{pfwt}_{s}(\{\sigma \in U: \tau \preccurlyeq \sigma\}) \geq 2^{-s|\tau|}$, then $\tau \in U$. Under reasonable assumptions, a c.e. set has a c.e. $s$-closed superset with almost the same prefix-free $s$-weight:

Lemma 2.1. Let $r \in[0,1]$ be rational and $R \subseteq 2^{<\omega}$ c.e. For every $\varepsilon>0$, there is an $r$-closed c.e. set $U \supseteq R$ such that $\operatorname{pfwt}_{r}(U) \leq \operatorname{pfwt}_{r}(R)+\varepsilon$.

Proof. We may assume that $\varepsilon$ is rational. Let $U \subseteq 2^{<\omega}$ be the closure of $R$ under the requirement that for every $\tau \in 2^{<\omega}$, if there is a finite prefix-free set $S \subseteq U$ of extensions of $\tau$ such that $\operatorname{pfwt}_{r}(S)>2^{-r|\tau|}-2^{-2|\tau|-1} \varepsilon$, then $\tau \in U$. It is easy to see that $U$ is c.e. and $r$-closed. Now let $T \subseteq U$ be a finite prefix-free set. By recursively replacing each $\tau \in T \backslash R$ with the finite prefix-free set of extensions that resulted in its inclusion in $U$, we get a finite prefix-free $T^{\prime} \subseteq R$ such that

$$
\mathrm{wt}_{r}(T)<\mathrm{wt}_{r}\left(T^{\prime}\right)+\sum_{\tau \in 2<\omega} 2^{-2|\tau|-1} \varepsilon \leq \operatorname{pfwt}_{r}(R)+\varepsilon .
$$

Therefore, $\operatorname{pfwt}_{r}(U) \leq \operatorname{pfwt}_{r}(R)+\varepsilon$.

\footnotetext{
${ }^{3}$ This definition differs slightly from Nies [19], where the sum is required to be $\leq 1$.
} 
If $W \subseteq 2^{<\omega}$, define $W^{n}$ for all $n \in \omega$ as follows. Let $W^{0}=\{\lambda\}$, where $\lambda$ is the empty sequence. Let $W^{n+1}=\left\{\sigma \tau: \sigma \in W^{n}\right.$ and $\left.\tau \in W\right\}$. The next lemma is completely routine.

Lemma 2.2. Let $s \in[0,1]$ and let $W \subseteq 2^{<\omega}$ be a c.e. set such that $\operatorname{wt}_{s}(W)<1$. If $X \in\left[W^{n}\right]$ for all $n \in \omega$, then $\operatorname{dim}(X) \leq s$.

Proof. Let $U=\bigcup_{n \in \omega} W^{n}$. Let $r>s$ be rational. Note that $\operatorname{wt}_{s}\left(W^{n}\right)=\mathrm{wt}_{s}(W)^{n}$ for all $n \in \omega$. Therefore, $\mathrm{wt}_{r}(U) \leq \mathrm{wt}_{s}(U) \leq \sum_{n \in \omega} \mathrm{wt}_{s}(W)^{n}<\infty$. This means that $\{\langle\sigma, r|\sigma|\rangle: \sigma \in U\}$ is a bounded request set, so $(\forall \sigma \in U)\left[K(\sigma) \leq^{+} r|\sigma|\right]$. Infinitely many prefixes of $X$ are in $U$, so $\operatorname{dim}(X) \leq r$. But $r>s$ was any rational number, so $\operatorname{dim}(X) \leq s$.

Proposition 2.3. (3) $\Rightarrow$ (4).

Proof. We prove the contrapositive, so assume that the $\Sigma_{1}^{0}$-covering property fails for $A \in 2^{\omega}$. Fix a counterexample: Let $W \subseteq 2^{<\omega}$ be an $A$-c.e. set and let $s, t \in[0,1]$ be real numbers such that $s<t$ and $\operatorname{wt}_{s}(W)<1$. Assume that there is no c.e. set $V$ such that $\mathrm{wt}_{t}(V)<1$ and $[W] \subseteq[V]$.

Fix a rational $r$ such that $s<r<t$. Consider a c.e. set $S$ such that $\operatorname{pfwt}_{r}(S)<1$. We claim that $[W] \nsubseteq[S]$. To see this, note that

$$
\begin{aligned}
\mathrm{wt}_{t}(S)=\sum_{n \in \omega}\left|S \cap 2^{n}\right| 2^{-t n}=\sum_{n \in \omega} \mid S & \cap 2^{n} \mid 2^{-r n} 2^{(r-t) n} \\
& \leq \sum_{n \in \omega} \operatorname{pfwt}_{r}(S) 2^{(r-t) n}<\sum_{n \in \omega} 2^{(r-t) n}<\infty .
\end{aligned}
$$

Since $\mathrm{wt}_{t}(S)<\infty$, there is an $m \in \omega$ such that $\mathrm{wt}_{t}\left(S \backslash 2^{\leq m}\right)<1-\operatorname{pfwt}_{r}(S)$. Since $S \cap 2^{\leq m}$ is finite, it is covered by a finite prefix-free subset $V_{0}$ of itself. Of course, $\mathrm{wt}_{t}\left(V_{0}\right) \leq \operatorname{pfwt}_{t}(S) \leq \operatorname{pfwt}_{r}(S)$. Let $V=V_{0} \cup\left(S \backslash 2^{\leq m}\right)$. Then $V$ is c.e., $[V]=[S]$, and $\mathrm{wt}_{t}(V)=\mathrm{wt}_{t}\left(V_{0}\right)+\mathrm{wt}_{t}(S \backslash 2 \leq m)<\operatorname{pfwt}_{r}(S)+\left(1-\operatorname{pfwt}_{r}(S)\right)=1$. By assumption, $[W] \nsubseteq[V]=[S]$.

Let $R=\left\{\sigma \in 2^{<\omega}: K(\sigma) \leq r|\sigma|\right\}$. So $R$ is a c.e. set and

$$
\operatorname{pfwt}_{r}(R) \leq \mathrm{wt}_{r}(R)=\sum_{\sigma \in R} 2^{-r|\sigma|} \leq \sum_{\sigma \in 2^{<\omega}} 2^{-K(\sigma)}<1 .
$$

Note that if $X \notin[R]$, then $\operatorname{dim}(X) \geq r$. By Lemma 2.1, there is an $r$-closed c.e. set $U \supseteq R$ such that $\operatorname{pfwt}_{r}(U)<1$.

We construct a sequence of strings $\left\{\sigma_{k}\right\}_{k \in \omega}$ such that for all $k \in \omega, \sigma_{k} \in W$, and $X=\sigma_{0} \sigma_{1} \sigma_{2} \cdots$ is not in $[U]$. Let $\tau_{k}=\sigma_{0} \cdots \sigma_{k-1}$, so in particular, $\tau_{0}=\lambda$. Since $\operatorname{pfwt}_{r}(U)<1$, we know that $\left[\tau_{0}\right] \nsubseteq[U]$. Now suppose that $\tau_{k}$ has been defined and $\left[\tau_{k}\right] \nsubseteq[U]$. Consider $U_{\tau_{k}}=\left\{\sigma \in 2^{<\omega}: \tau_{k} \sigma \in U\right\}$. Since $U$ is $r$-closed, $\operatorname{pfwt}_{r}\left(U_{\tau_{k}}\right) \geq 1$ would imply that $\tau_{k} \in U$. Therefore, $\operatorname{pfwt}_{r}\left(U_{\tau_{k}}\right)<1$, so by the discussion above, there is a $\sigma_{k} \in W$ such that $\left[\sigma_{k}\right] \nsubseteq\left[U_{\tau_{k}}\right]$. This means that $\left[\tau_{k+1}\right]=\left[\tau_{k} \sigma_{k}\right] \nsubseteq[U]$.

The construction ensures that $X \notin[U]$. Since $[R] \subseteq[U]$, we have $X \notin[R]$ and so $\operatorname{dim}(X) \geq r$. On the other hand, $X \in\left[W^{n}\right]$ for all $n \in \omega$, so $\operatorname{dim}^{A}(X) \leq s$ by Lemma 2.2 relativized to $A$. Therefore, $\operatorname{dim}^{A}(X) \leq s<r \leq \operatorname{dim}(X)$. Hence $A$ is not low for dimension.

We are ready to make the transition from the setting of infinite sequences to the world of finite strings. 
Proposition 2.4. $(4) \Rightarrow(5)$.

Proof. Assume that $A \in 2^{\omega}$ has the $\Sigma_{1}^{0}$-covering property. To prove that $A$ has the c.e. covering property, it is sufficient to consider rational numbers $s$ and $t$ with $0 \leq s<t<1$. Fix these, and let $r=\frac{s}{1-t+s}=t-\frac{(1-t)(t-s)}{1-t+s}$. Note that $s<r<t$. We will apply the $\Sigma_{1}^{0}$-covering property to the pair $s, r$, which gives us extra room when proving the c.e. covering property for $s, t$.

Let $\left\{S_{e}\right\}_{e \in \omega}$ be an effective list of all $A$-c.e. sets of strings (sufficiently universal to apply the Recursion Theorem below). Uniformly in $e \in \omega$, there is an $A$-c.e. set $\widehat{S}_{e} \subseteq S_{e}$ such that $\operatorname{wt}_{s}\left(\widehat{S}_{e}\right) \leq 2^{-e-1}$ and if $\operatorname{wt}_{s}\left(S_{e}\right) \leq 2^{-e-1}$, then $\widehat{S}_{e}=S_{e}$. Let $S=\bigcup_{e \in \omega} \widehat{S}_{e}$. Then

$$
\mathrm{wt}_{s}(S) \leq \sum_{e \in \omega} \mathrm{wt}_{s}\left(\widehat{S}_{e}\right)<\sum_{e \in \omega} 2^{-e-1}=1
$$

where the strict inequality follows from the fact that $S_{e}=\emptyset$ for some $e \in \omega$. By the $\Sigma_{1}^{0}$-covering property, there is a c.e. set $R \subseteq 2^{<\omega}$ such that $[S] \subseteq[R]$ and $\mathrm{wt}_{r}(R)<1$. This is our only use of the $\Sigma_{1}^{0}$-covering property.

Let $W \subseteq 2^{<\omega}$ be an $A$-c.e. set such that $\mathrm{wt}_{s}(W)<1$. Our goal is to find a c.e. set $V \supseteq W$ such that $\mathrm{wt}_{t}(V)<\infty$. We will build an auxiliary $A$-c.e. set $U$. By the Recursion Theorem, we may assume that the construction of $U$ has access to a parameter $e \in \omega$ such that $U=S_{e}$. As long as we ensure that $\operatorname{wt}_{s}(U) \leq 2^{-e-1}$, then $[U]=\left[\widehat{S}_{e}\right] \subseteq[R]$.

Before we proceed to the details, it will be helpful to sketch the construction of $V$. When a string $\sigma$ is enumerated into $W$, we will respond by putting a code string $\tau$ into $U$ and waiting for $[\tau]$ to be covered by $[R]$. Say that this happens because $\rho \preccurlyeq \tau$ goes into $R$. If $\rho$ and $\tau$ are approximately of the same length, then we can use the enumeration of $\rho$ as permission to put $\sigma$ into $V$. If $\rho$ is much shorter than $\tau$, then its enumeration gives us permission to try again by putting a new code string into $U$ for the sake of $\sigma$. Of course, it is possible that $[\tau]$ is covered by a collection of longer strings in $R$. To eliminate the need to consider this possibility explicitly, we replace $R$ by the set $Q$ of all strings that are covered by extensions in $R$. For $n \in \omega$, let $2^{\geq n}=\left\{\rho \in 2^{<\omega}:|\rho| \geq n\right\}$. Let

$$
Q=\left\{\tau \in 2^{<\omega}:[\tau] \subseteq\left[R \cap 2^{\geq|\tau|}\right]\right\} .
$$

It is clear that $[Q]=[R]$. We want to show that $\mathrm{wt}_{r}(Q)<\infty$. Consider the contribution that a string $\rho \in R$ makes to $\operatorname{wt}_{r}(Q)$. For every $\tau \preccurlyeq \rho$ that ends up in $Q$, we could attribute $2^{|\tau|-|\rho|}$ of its $r$-weight $2^{-r|\tau|}$ to $\rho$. In this way, the total cost of $\tau \in Q$ is split up between the strings from $R$ that cover it. So

$$
\mathrm{wt}_{r}(Q) \leq \sum_{\rho \in R} \sum_{m \leq|\rho|} 2^{m-|\rho|} 2^{-r m} \leq \sum_{\rho \in R} O(1) 2^{-r|\rho|}=O(1) \mathrm{wt}_{r}(R)<\infty .
$$

We will also use the fact that

$$
\mu[Q]=\mu[R] \leq \sum_{\rho \in R} 2^{-|\rho|} \leq \sum_{\rho \in R} 2^{-r|\rho|}=\mathrm{wt}_{r}(R)<1 .
$$

Fix $c \in \omega$ large enough that $\mu[Q] \leq 1-2^{-c}$ and $2^{-s c} \leq 2^{-e-3}$. Fix $d \in \omega$ large enough that $2^{-d} \mathrm{wt}_{r}(Q) \leq 2^{-e-2}$. 
Let $\left\{\rho_{k}\right\}_{k \in \omega}$ be a computable enumeration of $Q$ and let $Q_{k}=\left\{\rho_{i}\right\}_{i<k}$. We dynamically assign code strings. At each stage $k \in \omega$, we have a (computable) injection $\tau_{k}: 2^{<\omega} \rightarrow 2^{<\omega}$ such that for every $\sigma \in 2^{<\omega}$

- $\left|\tau_{k}(\sigma)\right|=|\sigma|+c$,

- no prefix of $\tau_{k}(\sigma)$ is in $Q_{k}$, and

- if $\tau_{k}(\sigma) \neq \tau_{k+1}(\sigma)$, then there is a prefix of $\tau_{k}(\sigma)$ in $Q_{k+1}$.

Note that it is always possible to reassign strings: $\mu[Q]<1-2^{-c}$ implies that, for each $n \in \omega$, there are at least $2^{-c} 2^{n+c}=2^{n}$ usable strings of length $n+c$.

The purpose of the code strings should be clearer if we describe how $Q$ is decoded to produce a c.e. set $V \subseteq 2^{<\omega}$. If $\rho \in 2^{<\omega}$ enters $Q$ at stage $k, \tau_{k}(\sigma) \succcurlyeq \rho$, and $\left|\tau_{k}(\sigma)\right|<\frac{r|\rho|+d}{s}$, then put $\sigma$ into $V$. Note that several strings may enter $V$ in response to a single $\rho$ entering $Q$. Despite this, we can show that $\operatorname{wt}_{t}(V)<\infty$. Consider $\rho \in Q$ with $n=|\rho|$. The contribution of $\rho$ to $\mathrm{wt}_{t}(V)$ is at most

$$
\sum_{m<\frac{r n+d}{s}-n} 2^{m} 2^{-t(n+m-c)} \leq O(1) 2^{(1-t)\left[\frac{r n+d}{s}-n\right]-t n}=O(1) 2^{-r n},
$$

where the last equality follows from the fact that $(1-t)\left[\frac{r}{s}-1\right]-t=-r$. (Indeed, this is the reason for our choice of $r$.) Therefore, $\mathrm{wt}_{t}(V) \leq \sum_{\rho \in Q} O(1) 2^{-r|\rho|}=$ $O(1) \mathrm{wt}_{r}(Q)<\infty$.

We are now ready to build $U$. Recall that $U \subseteq 2^{<\omega}$ is the $A$-c.e. set that we are constructing, and that if $\mathrm{wt}_{s}(U) \leq 2^{-e-1}$, then $[U] \subseteq[R]=[Q]$. The goal of the construction is to ensure that $V \supseteq W$. Let $\left\{\sigma_{j}\right\}_{j \in \omega}$ be an $A$-computable enumeration of $W$. Let $k_{0}=0$. At stage $k_{i}$ of the construction, let $\sigma_{j}$ be the first element of the enumeration of $W$ that we have not yet successfully coded. Put $\tau=\tau_{k_{i}}\left(\sigma_{j}\right)$ into $U$ and wait for the first stage $k_{i+1}$ at which some $\rho \preccurlyeq \tau$ enters $Q$. If $|\tau|<\frac{r|\rho|+d}{s}$, then we have successfully coded $\sigma_{j}$, since in that case, $\sigma_{j} \in V$. At stage $k_{i+1}$, we either attempt again to code $\sigma_{j}$, or if we were successful, attempt to code $\sigma_{j+1}$. This completes the construction.

We must prove that $\operatorname{wt}_{s}(U) \leq 2^{-e-1}$ without assuming that $[U] \subseteq[Q]$. Note, however, that if $[U] \nsubseteq[Q]$, then there is some final stage $k_{i}$ at which we enumerate a string into $U$, and then we wait forever for a stage $k_{i+1}$. So there is at most one string $\hat{\tau} \in U$ with $[\hat{\tau}] \nsubseteq[Q]$. Since $|\hat{\tau}| \geq c, 2^{-s|\hat{\tau}|} \leq 2^{-s c} \leq 2^{-e-3}$.

For all remaining $\tau \in U$, some prefix of $\tau$ is necessarily enumerated into $Q$. The total cost of all of the $\tau$ that are put into $U$ that result in a successful coding is $\sum_{\sigma \in W} 2^{-s(|\sigma|+c)}=2^{-s c} \mathrm{wt}_{s}(W)<2^{-s c} \leq 2^{-e-3}$. For every $\tau$ other than $\hat{\tau}$ that is put into $U$ but does not result in a successful coding, there is a corresponding $\rho \in Q$ such that $|\tau| \geq \frac{r|\rho|+d}{s}$. Hence the total cost of these $\tau$ is at most

$$
\sum_{\rho \in Q} 2^{-s\left(\frac{r|\rho|+d}{s}\right)}=2^{-d} \mathrm{wt}_{r}(Q) \leq 2^{-e-2} .
$$

Therefore, $\operatorname{wt}_{s}(U) \leq 2^{-e-3}+2^{-e-3}+2^{-e-2}=2^{-e-1}$. This means that every $\tau$ we put into $U$ eventually has a prefix in $Q$. Furthermore, we cannot put more than $2^{\left|\sigma_{j}\right|+c}$ strings into $U$ for the sake of $\sigma_{j}$, so it must eventually be successfully coded. Hence $W \subseteq V$. 


\section{LOWISHNESS FOR $K$}

The next goal is to prove that if $A \in 2^{\omega}$ is low for dimension, then it is lowish for $K$. An easy lemma shows that the c.e. covering property gives us a connection between $K$ and $K^{A}$. From there we show that (6) holds for $A$, meaning that $K(\sigma)-K^{A}(\sigma)$ is asymptotically small relative to $|\sigma|$. Finally, we prove that $A$ is lowish for $K$, in other words, that $K(\sigma)-K^{A}(\sigma)$ is asymptotically small even relative to $K(\sigma)$.

Lemma 3.1. Assume that $A$ has the c.e. covering property and that $0 \leq s<t \leq 1$. Then $\left(\forall \sigma \in 2^{<\omega}\right)\left[K^{A}(\sigma) \leq s|\sigma| \Rightarrow K(\sigma) \leq{ }^{+} t|\sigma|\right]$, where the constant does not depend on $\sigma$.

Proof. Let $W=\left\{\sigma: K^{A}(\sigma) \leq s|\sigma|\right\}$. So $W$ is $A$-c.e. and

$$
\mathrm{wt}_{s}(W)=\sum_{\sigma \in W} 2^{-s|\sigma|} \leq \sum_{\sigma \in W} 2^{-K^{A}(\sigma)}<1 .
$$

Therefore, there is a c.e. set $V \supseteq W$ such that $\mathrm{wt}_{t}(V)<\infty$. The latter condition implies that $U=\{\langle\sigma, t|\sigma|\rangle: \sigma \in V\}$ is a bounded request set, so for every $\sigma \in V$, we have $K(\sigma) \leq^{+} t|\sigma|$.

Proposition 3.2. $(5) \Rightarrow(6)$.

Proof. Assume that (6) fails, so there are a rational $r<0$ and a sequence of distinct strings $\left\{\sigma_{i}\right\}_{i \in \omega}$ such that $(\forall i)\left[K^{A}\left(\sigma_{i}\right)-K\left(\sigma_{i}\right)<r\left|\sigma_{i}\right|\right]$. Let $\ell=\liminf _{i \rightarrow \infty} K^{A}\left(\sigma_{i}\right) /\left|\sigma_{i}\right|$. Clearly, $\ell \in[0,1]$. Note that

$$
\ell=\liminf _{i \rightarrow \infty} \frac{K^{A}\left(\sigma_{i}\right)}{\left|\sigma_{i}\right|} \leq \liminf _{i \rightarrow \infty} \frac{K\left(\sigma_{i}\right)+r\left|\sigma_{i}\right|}{\left|\sigma_{i}\right|} \leq 1+r<1 .
$$

Take $s$ and $t$ such that $\ell<s<t<1$ and $\ell-t>r$. Thin $\left\{\sigma_{i}\right\}_{i \in \omega}$ to a subsequence $\left\{\tau_{i}\right\}_{i \in \omega}$ such that $(\forall i)\left[K^{A}\left(\tau_{i}\right) \leq s\left|\tau_{i}\right|\right]$. By Lemma $3.1, K\left(\tau_{i}\right) \leq^{+} t\left|\tau_{i}\right|$. This means that $\lim \sup _{i \rightarrow \infty} K\left(\tau_{i}\right) /\left|\tau_{i}\right| \leq t$. Since $\left\{\tau_{i}\right\}_{i \in \omega}$ is a subsequence of $\left\{\sigma_{i}\right\}_{i \in \omega}$, we have $\liminf _{i \rightarrow \infty} K^{A}\left(\tau_{i}\right) /\left|\tau_{i}\right| \geq \ell$. Therefore,

$$
\liminf _{i \rightarrow \infty} \frac{K^{A}\left(\tau_{i}\right)-K\left(\tau_{i}\right)}{\left|\tau_{i}\right|} \geq \liminf _{i \rightarrow \infty} \frac{K^{A}\left(\tau_{i}\right)}{\left|\tau_{i}\right|}-\limsup _{i \rightarrow \infty} \frac{K\left(\tau_{i}\right)}{\left|\tau_{i}\right|} \geq \ell-t>r .
$$

This contradicts the fact that $K^{A}\left(\tau_{i}\right)-K\left(\tau_{i}\right)<r\left|\tau_{i}\right|$ for all $i \in \omega$.

Proposition 3.3. (6) $\Rightarrow(7)$.

Proof. We prove the contrapositive. Assume that (7) fails, so there is an $\varepsilon>0$ such that $K^{A}(\sigma)<(1-\varepsilon) K(\sigma)$ for infinitely many $\sigma$. This says that there are infinitely many $\sigma$ that $A$ can compress better by a multiplicative factor of $K(\sigma)$. To prove the failure of (6), we need to show that $A$ compresses infinitely many $\eta$ better by a multiplicative factor of $|\eta|$. If a witness $\sigma$ to the failure of (7) has low complexity, then it might not serve as a witness to the failure of (6). The idea is to replace $\sigma$ with a short $\eta$ such that $U(\eta)=\sigma$. The choice of $\eta$ will be made so that it is essentially a compressed witness to the failure of (7), hence also to the failure of (6). In particular, we will find infinitely many $\eta$ for which $K^{A}(\eta)-K(\eta) \leq^{+}-\varepsilon|\eta|$, which is sufficient.

Let $U^{X}$ denote the universal prefix-free machine with oracle $X$. We define a prefix-free machine $M^{A}$ relative to $A$ as follows. If $U^{A}(\rho) \downarrow=\sigma$ and $k \in \omega$, let 
$\tau=\rho 0^{k} 1$ and search for a string $\eta$ such that $U(\eta)=\sigma$ and $(1-\varepsilon)|\eta| \leq|\tau|$. If such an $\eta$ is found, let $M^{A}(\tau)=\eta$. The fact that $U^{A}$ is prefix-free implies that $M^{A}$ is prefix-free.

Take $\sigma$ for which $K^{A}(\sigma)<(1-\varepsilon) K(\sigma)$. Let $\rho$ be a minimal $U^{A}$-program for $\sigma$ and let $\widehat{\eta}$ be a minimal $U$-program for $\sigma$. By assumption, $|\rho|<(1-\varepsilon)|\widehat{\eta}|$. Let $k \in \omega$ be minimal such that $(1-\varepsilon)|\widehat{\eta}| \leq|\rho|+k+1$ and let $\tau=\rho 0^{k} 1$. This ensures that $M^{A}(\tau)$ converges, though possibly not to $\widehat{\eta}$. Let $\eta=M^{A}(\tau)$. Our choice of $k$ ensures that $|\tau|-1<(1-\varepsilon)|\widehat{\eta}| \leq(1-\varepsilon)|\eta| \leq|\tau|$, from which it follows that $|\eta|=^{+}|\widehat{\eta}|$. Also note that $|\widehat{\eta}|=K(\sigma)=K(U(\eta)) \leq^{+} K(\eta)$. Putting everything together, we obtain

$$
K^{A}(\eta)-K(\eta) \leq^{+}|\tau|-|\widehat{\eta}| \leq^{+}(1-\varepsilon)|\eta|-|\eta|=-\varepsilon|\eta| .
$$

\section{LOWISHNESS FOR RANDOMNESS AND LOWNESS FOR DIMENSION $s \in(0,1]$}

We have almost completed the proof of the main theorem. All that remains it to show that the following properties are also equivalent to lowness for dimension.

(1) $A$ is lowish for randomness, i.e., every Martin-Löf random sequence has dimension 1 relative to $A$,

(2) $A$ is low for some dimension $s \in(0,1]$.

It is easy to see that both properties are implied by lowness for dimension. In particular, we have already noted that $(3) \Rightarrow(2)$.

Proposition 4.1. $(2) \Rightarrow(1)$.

Proof. Assume that $A \in 2^{\omega}$ is low for dimension $s \in(0,1]$. Given a Martin-Löf random sequence $X \in 2^{\omega}$, we will spread $X$ out to create a sequence $s[X]$ of dimension $s$. We will then observe that $\operatorname{dim}^{A}(s[X])=s$ implies that $\operatorname{dim}^{A}(X)=1$.

For $Z \in 2^{\omega}$, the spread out version of $Z$ is $s[Z]=\{\lfloor n / s\rfloor: Z(n)=1\}$. We want to show that $K\left(s\left[1^{\omega}\right]\lceil n)\right.$ is $O(\log n)$. Fix $n \in \omega$ and let $r$ be the maximum value of $\lfloor m / s\rfloor / m$ for all $m<s n$. Then $r$ is a rational approximation of $1 / s$ that is good enough to determine $s\left[1^{\omega}\right]\lceil n$. To see this, consider any $m<s n$. Then $\lfloor r m\rfloor \geq\lfloor\lfloor m / s\rfloor / m \cdot m\rfloor=\lfloor m / s\rfloor$. But it also follows from the definition that $r \leq 1 / s$, so $\lfloor r m\rfloor \leq\lfloor m / s\rfloor$. Therefore, $\lfloor r m\rfloor=\lfloor m / s\rfloor$ for all such $m$. So $s\left[1^{\omega}\right]\lceil n$ can be computed uniformly from $r, n$ and $\max \{m: m<s n\}$. But $r$ is a ratio of natural numbers less than $n$, so $K\left(s\left[1^{\omega}\right]\lceil n)\right.$ is $O(\log n)$.

Now note that $K\left(s[Z]\lceil n)\right.$ differs from $K\left(Z\lceil\lfloor s n\rfloor)\right.$ by at most $K\left(s\left[1^{\omega}\right]\lceil n)+O(1)\right.$ because all we need to know to translate between the two strings is the location of the bits of $Z$ in the spread out version. Therefore

$$
\begin{aligned}
\operatorname{dim}(s[Z])=\liminf _{n \rightarrow \infty} \frac{K(s[Z]\lceil n)}{n}=\liminf _{n \rightarrow \infty} \frac{K(Z\lceil\lfloor s n\rfloor)+O(\log n)}{n} \\
=s \liminf _{n \rightarrow \infty} \frac{K(Z\lceil\lfloor s n\rfloor)}{s n}+\lim _{n \rightarrow \infty} \frac{O(\log n)}{n}=s \operatorname{dim}(Z) .
\end{aligned}
$$

Similarly, $\operatorname{dim}^{A}(s[Z])=s \operatorname{dim}^{A}(Z)$ for all $Z \in 2^{\omega}$.

To finish the proof, let $X \in 2^{\omega}$ be any Martin-Löf random sequence. Then $\operatorname{dim}(s[X])=s$, which implies that $\operatorname{dim}^{A}(s[X])=s$. $\operatorname{Sodim}^{A}(X)=\frac{1}{s} \operatorname{dim}^{A}(s[X])=$ 1. This proves that $A$ is lowish for randomness.

Proposition 4.2. (1) $\Rightarrow(3)$. 
Proof. We prove the contrapositive, so assume that $A \in 2^{\omega}$ is not low for dimension. Therefore, there is an $X \in 2^{\omega}$ and a rational $t>0$ such that $4 t \leq \operatorname{dim}(X)-$ $\operatorname{dim}^{A}(X)$. The idea is to code $X$ compactly into a random sequence $Z \in 2^{\omega}$ and use the fact that $A$ is better at compressing $X$ to prove that $\operatorname{dim}^{A}(Z)<1$.

Fix a functional $\Phi$ and let $W=\left\{\langle\sigma, d\rangle: d>t|\sigma|-\log \mu\left(\Phi^{-1}[\sigma]\right)\right\}$, where $\Phi^{-1}[\sigma]=\left\{Y: \Phi^{Y}\lceil|\sigma|=\sigma\}\right.$. Then $W$ is a c.e. set and

$$
\begin{aligned}
\sum_{\langle\sigma, d\rangle \in W} 2^{-d} & \leq \sum_{\sigma \in 2^{<\omega}} 2^{-t|\sigma|} \mu\left(\Phi^{-1}[\sigma]\right) \\
& =\sum_{n \in \omega} 2^{-t n} \sum_{\sigma \in 2^{n}} \mu\left(\Phi^{-1}[\sigma]\right) \leq \sum_{n \in \omega} 2^{-t n}<\infty,
\end{aligned}
$$

where we use the fact that if $\sigma$ and $\tau$ are incomparable strings, then $\Phi^{-1}[\sigma] \cap$ $\Phi^{-1}[\tau]=\emptyset$. Thus $W$ is a bounded request set, so there is a $c \in \omega$ such that for all $\sigma \in 2^{<\omega}$, we have $K(\sigma) \leq t|\sigma|-\log \mu\left(\Phi^{-1}[\sigma]\right)+c$. Therefore, $\mu\left(\Phi^{-1}[\sigma]\right) \leq$ $2^{t|\sigma|-K(\sigma)+c}$.

The next step is to code $X$ into a Martin-Löf random sequence. Kučera [13] and Gács [8] both proved that every sequence is computable from a Martin-Löf random. We need the version due to Gács, who proved that for any sequence $X$, there are a Martin-Löf random $Z$ and a functional $\Phi$ such that $\Phi^{Z}=X$ with use $\varphi(n)=n+o(n)$. We may assume that $\varphi(n)=2 n$, so for any $n$,

$$
Z\left\lceil 2 n \in U_{n}:=\left\{\rho \in 2^{2 n}: \Phi^{\rho}\lceil n=X\lceil n\} .\right.\right.
$$

From above, $\mu\left(\Phi^{-1}[X\lceil n]) \leq 2^{t n-K(X \nmid n)+c}\right.$ for some $c \in \omega$. So $U_{n}$ has size at most $2^{2 n+t n-K(X\lceil n)+c}$ and is uniformly c.e. in $X\lceil n$. We can code $Z\lceil 2 n$ using a code for $X\left\lceil n\right.$ and an index within $U_{n}$, therefore

$$
K^{A}\left(Z\lceil 2 n) \leq K^{A}(X\lceil n)+(2 n+t n-K(X\lceil n))+O(\log n) .\right.
$$

Here we use that if $m \leq 2^{2 n+t n-K(X \nmid n)+c}$, then $K(m) \leq^{+}(2 n+t n-K(X\lceil n))+$ $2 \log (2 n+t n-K(X\lceil n))$, and $\log (2 n+t n-K(X\lceil n)) \leq \log (2 n+t n)=O(\log n)$. By assumption, there are infinitely many $n$ such that $K\left(X\lceil n)-K^{A}(X\lceil n)>3 t n\right.$. For each such $n$, we have $K^{A}\left(Z\lceil 2 n) \leq 2 n-2 t n+O(\log n)\right.$, so $\operatorname{dim}^{A}(Z) \leq 1-t$. This proves that $A$ is not lowish for randomness.

\section{A PERFECT CLASS OF LOW FOR DIMENSION SETS}

As we mentioned in the introduction, only countably many sets are low for randomness, but continuum many are low for dimension. In this section, we prove the latter fact by constructing a perfect $\Pi_{1}^{0}$-class containing only lowish for $K$ sequences. This result was also recently proved by Herbert [9]. He built a perfect $\Pi_{1}^{0}$-class $Q \subseteq 2^{\omega}$ of sequences with finite self-information (see Section 7) and observed that, as a consequence of his construction, every element of $Q$ is low for dimension. His $\Pi_{1}^{0}$-class has another interesting property: every sequence is computable from the join of two elements of $Q$. Compare this to the fact that the join of two low for random sequences is also low for random (as proved for $K$-trivial sequences by Downey, Hirschfeldt, Nies and Stephan [7]).

Theorem 5.1. There is a perfect $\Pi_{1}^{0}$-class $P \subseteq 2^{\omega}$ such that every $A \in P$ is lowish for $K$. 
Proof. We construct a computable sequence $T_{s}: 2^{<\omega} \rightarrow 2^{<\omega}$ of partial function trees with finite domain such that $T(\sigma)=\lim _{s \rightarrow \infty} T_{s}(\sigma)$ is a total function. Our $\Pi_{1}^{0}$-class will be $P=[T]$, the set of infinite paths through $T .{ }^{4}$ We use $\sigma$ to denote strings in the domain of $T_{s}$, and $\tau$ to denote strings in the image of $T_{s}$. We let $U$ be the universal prefix-free oracle machine and we write $U^{\tau}$ for both the partial function and its domain. We use $\eta$ to denote strings in $U^{\tau}$. If $\sigma$ has positive length, then $\sigma^{-}$denotes $\sigma\lceil(|\sigma|-1)$.

Parameters: We say that $\tau$ is on $T_{s}$ if $\tau \preccurlyeq T_{s}(\sigma)$ for some $\sigma$ in the domain of $T_{s}$. For every $s \in \omega$, we keep $T_{s}(\lambda)=\lambda$ and $T_{s}(\sigma) \succcurlyeq T_{s-1}(\sigma)$ if both converge. If $T_{s}(\sigma) \succ T_{s-1}(\sigma)$, we say that $\sigma$ is moved at stage $s$. At every stage, at most one $\sigma$ is moved. If $\sigma$ is moved at stage $s$, then $T_{s}\left(\sigma^{\prime}\right) \uparrow$ for every $\sigma^{\prime} \succ \sigma$. We say that such $\sigma^{\prime}$ are initialized. For convenience, we require that for every $\sigma$ and $s, T_{s}(\sigma 0) \downarrow$ iff $T_{s}(\sigma 1) \downarrow$. To ensure that $[T]$ is a (perfect) $\Pi_{1}^{0}$-class, we also require that every leaf of $T_{s+1}$ extends a leaf of $T_{s}$. That is, if $\sigma$ is moved at a stage $s$ then we require that the new value $T_{s}(\sigma)$ extends a leaf of $T_{s-1}$.

We will make $T(X)=\bigcup_{n \in \omega} T\left(X\lceil n)\right.$ lowish for $K$ for every $X \in 2^{\omega}$. We let

$$
r_{n}=(n+2) /(n+1)
$$

for $n \in \omega$. We want to ensure that for every $X \in[T]$ and $n \in \omega$, and for almost every $\eta$ in the domain of $U^{X}$, we have that $K\left(U^{X}(\eta)\right) \leq{ }^{+} r_{n}|\eta|$. This would imply that every $X \in[T]$ is lowish for $K$ (see Lemma 5.4).

We enumerate a c.e. set $L$ consisting of sets of triples $\left\langle\eta, \tau, r_{n}\right\rangle$ (called requests) such that $\eta, \tau \in 2^{<\omega}$ and $n \in \omega$. The intention is that for each such request, we have $\eta \in U^{\tau}$ and we want $K\left(U^{\tau}(\eta)\right)$ to have complexity $\leq^{+} r_{n}|\eta|$. Such $L$-requests have to be made for each $\tau$ on $T$, and the entire construction will involve a careful accounting to keep the total weight of $L$ finite. Here we define

$$
\operatorname{wt}(L)=\sum_{\langle\eta, \tau, r\rangle \in L} 2^{-r|\eta|} .
$$

As long as $\operatorname{wt}(L)<\infty$, we can build a bounded request set from $L$ so that if $\langle\eta, \tau, r\rangle \in L$, then $K\left(U^{\tau}(\eta)\right) \leq^{+} r|\eta|$.

Viewed as a domain instead of an operator, we will assume that $U^{\tau}$ is a uniformly computable finite set of strings that increases with respect to $\tau$. As usual, $U^{X}=$ $\bigcup_{\tau \prec X} U^{\tau}$ is infinite and has weight less than 1 for each $X \in 2^{\omega}$. For convenience, we will also assume that $\left|U^{\tau 0} \backslash U^{\tau}\right|=1$ (which we can ensure by modifying the uses of computations). We order $2^{<\omega}$ first by length, then lexicographically, i.e., $\lambda<0<1<00<\cdots$. Associated with each $\sigma$ is a c.e. set $\operatorname{Bin}_{\sigma}$ that we build during the construction. This set will contain certain $L$-requests. The aim is to ensure that every $L$-request $\left\langle\eta, \tau, r_{n}\right\rangle$ issued during the construction will fall into $\operatorname{Bin}_{\sigma}$ for some $\sigma$. Intuitively, this means that we will charge the weight of this particular request to the node $\sigma$. The entire construction will attempt to keep the total weight in each $\operatorname{Bin}_{\sigma}$ small so that we can show that $\operatorname{wt}(L)<\infty$. We also maintain counters $i_{\sigma}$ for each $\sigma \in 2^{<\omega}$. These record the number of times that $\sigma$ has been initialized so far (initialization is formally defined in the construction).

\footnotetext{
${ }^{4}$ Note that $[T]$ is a closed subset of $2^{\omega}$. This notation should not be confused with our use of $[W]$ to mean the open set generated by $W \subseteq 2^{<\omega}$.
} 
For every $i \in \omega$, we define the computable sequence $t_{\sigma, i}$ inductively on $|\sigma|$ as follows:

$$
\begin{aligned}
& t_{\lambda, i}=0, \\
& t_{\sigma, i}=\left\langle\frac{t_{\sigma^{-}, i}}{r_{|\sigma|}-1}, \frac{\sigma-\log \left(1-2^{1-r_{|\sigma|}}\right)+5}{r_{|\sigma|}-1}, i, \sigma\right\rangle, \text { if }|\sigma|>0 .
\end{aligned}
$$

Here we identify $\sigma \in 2^{<\omega}$ with its code number and all fractions are rounded up, if necessary. This seemingly bizarre choice for $t_{\sigma, i}$ is simply to ensure that certain sums converge and will be made clear during the verification. We prefer to take $\langle\cdot\rangle$ rather than $\max \{\cdot\}$ in the definition for $t_{\sigma, i}$ because we want the quantity $t_{\sigma, i}$ to be strictly increasing in $i$.

During the construction, when we refer to $t_{\sigma}$ at a stage $s$, we mean the value of $t_{\sigma, i_{\sigma}}$ evaluated at stage $s$. We sometimes write $t_{\sigma}[s]$. Each $t_{\sigma}$ represents a threshold value for $\sigma$.

During the construction, if we see some $\eta$ in $U^{\tau}$ for some $\tau$ on $T$, then we must issue a description for $U^{\tau}(\eta)$. Fortunately for us, we are allowed to issue a description of length $r|\eta|$ for some $r>1$, and so we would use up $2^{-r|\eta|}$ much measure to describe $U^{\tau}(\eta)$ compared to $2^{-|\eta|}$ for the opponent. It might appear that we have much more measure to play with compared to the opponent, but remember that the opponent has used up $2^{-|\eta|}$ much measure in the oracle machine $U^{\tau}$. This means that he could repeat this by adding $\eta$ to $U^{\tau_{1}}, U^{\tau_{2}}, \cdots, U^{\tau_{k}}$ for a large number $k$ (remember that our $\Pi_{1}^{0}$-class has to be perfect, so we must allow an antichain of arbitrarily large size to be extendible in our $\Pi_{1}^{0}$-class). So the opponent has really only used up $2^{-|\eta|}$ along each oracle, but we would have to use up $k \cdot 2^{-r|\eta|}$, which could be very large. We can, of course, counter this by picking a large $r$, but we must eventually use only small values of $r$ to ensure that each path is lowish for $K$, and so such a naive strategy will not succeed.

The solution to this problem is to block the accumulation of measure by the opponent as described above. We assign a threshold value $t_{\sigma}$ to each node $\sigma$. When we see new strings added to $U^{T\left(\sigma^{\prime}\right)} \backslash U^{T(\sigma)}$ with very small weight, we will issue requests for the new strings and charge the cost of these requests to $\sigma^{\prime}$. If these new strings all have weights smaller than the threshold values $t_{\sigma 0}$ and $t_{\sigma 1}$, then we will never move $T(\sigma), T(\sigma 0)$ or $T(\sigma 1)$, and the cost of describing these new strings are charged to extensions of $\sigma$. If, however, we see a new string $\eta$ added to, say, $U^{T\left(\sigma 0 \sigma^{\prime}\right)}$, that increases the cumulative weight above the threshold of $\sigma 0$ (i.e., $\left.\mu\left[U^{T\left(\sigma 0 \sigma^{\prime}\right)} \backslash U^{T(\sigma 0)}\right]>2^{-t_{\sigma 0}}\right)$, then we will charge the cost of describing $\eta$ to $\sigma$. We will also move $T(\sigma 0)$ to the position $T\left(\sigma 0 \sigma^{\prime}\right)$. Doing this will render (essentially) all of the $L$-requests previously made for oracles extending $T_{s}(\sigma 00)$ and $T_{s}(\sigma 01)$ wasted; however, if we are careful, these will have small weight, and we can reset all of the corresponding thresholds to be much larger.

Now observe that a request issued to describe $\eta$ is charged to $\sigma$ only if it was too costly for $\sigma 0$ (or $\sigma 1$ ), and in this case $\sigma 0$ will be moved so that $\eta$ is now in $U^{T(\sigma 0)} \backslash U^{T(\sigma)}$. Now the crucial point is that not too much weight can be charged to $\sigma$, because otherwise $U^{T(\sigma 0)} \backslash U^{T(\sigma)}$ would exceed the threshold $2^{-t_{\sigma}}$, and we would then move $\sigma$.

Barring the action of higher-priority nodes, $\sigma$ can only move in this way at most $2^{t_{\sigma}}$ many times, since each time it is moved, we have at least $2^{-t_{\sigma}}$ measure of new strings in $U^{T_{s+1}(\sigma)}$. Hence the total weight of garbage left in $L$ due to the movement 
of $\sigma$ in this way can be made small. This allows us to bound the overall weight of $L$.

Construction: At stage 0 , we start with $L=\emptyset, \operatorname{Bin}_{\sigma}=\emptyset$, and $i_{\sigma}=0$ for every $\sigma \in 2^{<\omega}$. Let $T_{0}(\lambda)=\lambda$. At stage $s>0$, let $\sigma$ be the least node such that $T_{s-1}(\sigma 0) \uparrow$ (and hence $\left.T_{s-1}(\sigma 1) \uparrow\right)$. We say that we act for $\sigma$. Let $\tau=T_{s-1}(\sigma)$, and let $\eta_{i} \in U^{\tau i} \backslash U^{\tau}$. We first process $\eta_{0}$ (before moving on, if necessary, to $\eta_{1}$, and then end stage $s$ ). To process $\eta_{0}$, there are two steps:

Step 1: Let $n \leq|\sigma|$ be largest such that $\left|\eta_{0}\right|>t_{\sigma \mid n}$. Note that $n$ exists as $t_{\lambda}=0$. We enumerate the request $\left\langle\eta_{0}, \tau 0, r_{n}\right\rangle$ into $L$.

Step 2: We now decide which prefix of $\sigma$ we will charge this request to. Let $\sigma_{0} \preccurlyeq \sigma$ be the minimal node such that $\mu\left[U^{\tau 0} \backslash U^{T_{s-1}\left(\sigma_{0}\right)}\right] \geq 2^{-t_{\sigma_{0}}}$. Note that $\sigma_{0} \neq \lambda$. If $\sigma_{0}$ exists, then we enumerate $\left\langle\eta_{0}, \tau 0, r_{n}\right\rangle$ into $\operatorname{Bin}_{\sigma_{0}^{-}}$. We move $\sigma_{0}$ by setting $T_{s}\left(\sigma_{0}\right)=\tau 0$, initializing every $\sigma^{\prime} \succ \sigma_{0}$, and letting $T_{s}$ copy $T_{s-1}$ everywhere else. Initializing $\sigma^{\prime}$ means that we increment $i_{\sigma^{\prime}}$ and adjust the value of the threshold $t_{\sigma^{\prime}}$. Note that initialization does not reset $\operatorname{Bin}_{\sigma^{\prime}}$. Go to the next stage. If $\sigma_{0}$ does not exist, then we set $T_{s}(\sigma 0)=\tau 0$ and put $\left\langle\eta_{0}, \tau 0, r_{n}\right\rangle$ into $\operatorname{Bin}_{\sigma}$.

If we did not stop the construction while processing $\eta_{0}$, we will move on and process $\eta_{1}$ in the same way.

Verification: It is clear that the sequence $T_{s}$ meets the conditions stated above (other than the totality of $T=\lim _{s \rightarrow \infty} T_{s}$, which we will verify below). Every $L$-request is also placed into $\operatorname{Bin}_{\sigma}$ for some $\sigma \in 2^{<\omega}$, so $\operatorname{wt}(L) \leq \sum_{\sigma \in 2<\omega} \operatorname{wt}\left(\operatorname{Bin}_{\sigma}\right)$. We need to show that $T$ is total, and each $\operatorname{wt}\left(\operatorname{Bin}_{\sigma}\right)$ is small. We first prove a crucial lemma:

Lemma 5.2. Consider $\sigma \in 2^{<\omega}$ and $s^{\prime}<s$ such that both $T_{s^{\prime}}(\sigma) \downarrow$ and $T_{s}(\sigma) \downarrow$. If $\sigma$ is not initialized strictly between $s^{\prime}$ and $s$, then $\mathrm{wt}\left[\operatorname{Bin}_{\sigma, s} \backslash \operatorname{Bin}_{\sigma, s^{\prime}}\right]<2^{\left(1-r_{|\sigma|}\right) t_{\sigma, s}+4}$.

Proof. Let $s^{\prime}<s_{0}<\cdots<s_{k} \leq s$ be all the stages at which $\operatorname{Bin}_{\sigma}$ receives new elements. Let $t=t_{\sigma, s}$, and note that $\sigma^{-}$is not moved at any of these stages. Consequently, it is easy to see that there are at most $2^{t}$ many $j \leq k$ for which $\sigma$ is moved at $s_{j}$. For each $i \leq k$, there are three possibilities for $s_{i}$ :

(i) $\sigma$ is moved at stage $s_{i}$. The only way for $\sigma$ to be moved and for $\operatorname{Bin}_{\sigma}$ to increase at stage $s_{i}$, is for us to act for $\sigma$ at stage $s_{i}$. Hence $\operatorname{Bin}_{\sigma}$ receives exactly one new request of the form $\left\langle\eta, \tau, r_{|\sigma|}\right\rangle$ when processing $\eta_{0}$ and $\sigma$ is moved when processing $\eta_{1}$. Since $|\eta|>t$, it follows that $2^{-r_{|\sigma|}|\eta|}<2^{-r_{|\sigma|} t}$.

(ii) We act for $\sigma$ at $s_{i}$ but $\sigma$ is not moved at stage $s_{i}$. In this case, $\operatorname{Bin}_{\sigma}$ receives two new requests of the form $\left\langle\eta, \tau, r_{|\sigma|}\right\rangle$, but note that this item (ii) will not apply again until $\sigma$ is again moved. As in (i), we have $2^{-r_{|\sigma|}|\eta|}<2^{-r_{|\sigma|} t}$ for each of the two requests.

(iii) Either $\sigma 0$ or $\sigma 1$ is moved (without loss of generality, assume the former). Then a request of the form $\left\langle\eta, \tau, r_{n}\right\rangle$ is added to $\operatorname{Bin}_{\sigma}$, where $|\eta|>t_{\sigma \sigma^{\prime}}$ and $n=\left|\sigma \sigma^{\prime}\right| \geq|\sigma|$. We claim that $2^{-r_{n}|\eta|}<2^{-|\eta|} 2^{\left(1-r_{|\sigma|}\right) t}$. If $\left|\sigma^{\prime}\right|=0$ then we are done as in case (i). Otherwise suppose that $\sigma \subset \sigma \sigma^{\prime}$. Now for any string $\alpha \neq \lambda$ and any $i$, we have that

$$
t_{\alpha, i}>\frac{t_{\alpha^{-}, i}}{r_{|\alpha|}-1}>\frac{r_{\left|\alpha^{-}\right|}-1}{r_{|\alpha|}-1} \cdot t_{\alpha^{-}, i}
$$


where the first inequality follows by the definition of $t$ and the second follows because $1<r_{\left|\alpha^{-}\right|}<2$. Applying this, together with the fact that $i_{\sigma \sigma^{\prime}} \geq i_{\sigma}$, we obtain $t_{\sigma \sigma^{\prime}}>\frac{r_{|\sigma|}-1}{r_{n}-1} t$. It follows that $|\eta|>\frac{r_{|\sigma|}-1}{r_{n}-1} t$, so our claim follows.

Now suppose that $s_{i}<s_{j}$ are two consecutive stages where $\sigma$ is moved (the same analysis below applies if either $s_{i}=s^{\prime}$ or $s_{j}=s$ ). We now account for how much weight is added to $\operatorname{Bin}_{\sigma}$ at those stages $s_{i_{0}}$, where $s_{i}<s_{i_{0}}<s_{j}$. (ii) contributes on at most one stage, and the sum of the contributions from (iii) is at $\operatorname{most} \sum_{\eta} 2^{-|\eta|} 2^{\left(1-r_{|\sigma|}\right) t}=2^{\left(1-r_{|\sigma|}\right) t} \cdot \sum_{\eta} 2^{-|\eta|}$, where the sum is taken over all strings $\eta$ in $U^{T(\sigma 0)} \backslash U^{T(\sigma)}$ or $U^{T(\sigma 1)} \backslash U^{T(\sigma)}$. Now clearly this sum $\sum_{\eta} 2^{-|\eta|}$ is less than twice the threshold value $2 \cdot 2^{-t}$, because otherwise one of the two $U^{T(\sigma 0)} \backslash U^{T(\sigma)}$ or $U^{T(\sigma 1)} \backslash U^{T(\sigma)}$ would get too heavy and we would be forced to move $\sigma$ before $s_{j}$. Thus we conclude that the sum of contributions from (iii) is at most $2 \cdot 2^{-t} 2^{\left(1-r_{|\sigma|}\right) t}=2^{-r_{|\sigma|} t+1}$.

Totaling the contribution of all the stages $s_{i}$, we have $\operatorname{wt}\left(\operatorname{Bin}_{\sigma, s} \backslash \operatorname{Bin}_{\sigma, s^{\prime}}\right) \leq$ $\left(2^{t}+1\right)((\mathrm{i})+(\mathrm{ii})+(\mathrm{iii})) \leq\left(2^{t}+1\right)\left(3 \cdot 2^{-r_{|\sigma|} t}+2^{-r_{|\sigma|} t+1}\right)<2^{\left(1-r_{|\sigma|}\right) t+4}$.

Lemma 5.3. For each $\sigma \in 2^{<\omega}$, we have $\mathrm{wt}\left(\operatorname{Bin}_{\sigma}\right)<2^{-\sigma}$. (Here we identify $\sigma$ with its code number).

Proof. Fix $\sigma \in 2^{<\omega}$. We do not add anything to $\operatorname{Bin}_{\sigma}$ at a stage $s \in \omega$ unless $T_{s-1}(\sigma) \downarrow$. Each time $\sigma$ is initialized, $t_{\sigma}$ is increased. To calculate an upper bound on wt $\left(\operatorname{Bin}_{\sigma}\right)$, we consider the stages $s_{0}<s_{1}<\cdots$ where $\sigma$ is initialized. The total weight added to $\operatorname{Bin}_{\sigma}$ for stages $s_{i}<s<s_{i+1}$, for every $i$, can be bounded by applying Lemma 5.2. This weight is at most

$$
\sum_{t \geq t_{\sigma, 0}} 2^{\left(1-r_{|\sigma|}\right) t+4}=\frac{2^{\left(1-r_{|\sigma|}\right) t_{\sigma, 0}+4}}{1-2^{\left(1-r_{|\sigma|}\right)}} \leq 2^{-\sigma-1} .
$$

The first equality follows by applying the sum of a geometric series, while the second inequality follows from the fact that $t_{\sigma, 0}<\frac{\sigma-\log \left(1-2^{1-r|\sigma|}\right)+5}{r_{|\sigma|-1}}$.

On the other hand, if anything is added to $\operatorname{Bin}_{\sigma}$ at stage $s_{i}$, then we must have acted for $\sigma$ at stage $s_{i}$, and the request was added to $\operatorname{Bin}_{\sigma}$ during the processing of $\eta_{0}$. This is at most $2^{-r_{|\sigma|} t_{\sigma}\left[s_{i}\right]}<2^{-t_{\sigma}\left[s_{i}\right]}$. So the sum of the weights of stuff added at all $s_{i}$ is bounded above by

$$
\sum_{i} 2^{-t_{\sigma, i}}<2^{-t_{\sigma, 0}+1}<2^{-\sigma-1}
$$

Therefore adding both parts together we have $\operatorname{wt}\left(\operatorname{Bin}_{\sigma}\right)<2^{-\sigma}$.

The previous lemma tells us that $\operatorname{wt}(L) \leq 1$. It follows by a straightforward induction on $|\sigma|$ that each $\sigma$ is moved finitely often and each $\sigma$ is initialized finitely often. Hence $T$ is total. It is routine to verify (by induction on $s$ ) that for every $s$, every $\tau$ on $T_{s}$, and every $\eta \in U^{\tau} \backslash U^{\tau^{-}}$, we have $\langle\eta, \tau, r\rangle$ in $L$ for some $r$.

Lemma 5.4. For each $X \in 2^{\omega}, T(X)$ is lowish for $K$.

Proof. Let $X \in 2^{\omega}$, and fix an $n \in \omega$. We want to show that for almost every $\eta \in$ $U^{T(X)}$, we enumerate $\left\langle\eta, \tau, r_{m}\right\rangle$ into $L$ for the sake of $U^{T(X)}$, for some $m \geq n$. Let $s$ be a stage where both $t_{X \uparrow n}$ and $T_{s}\left(X\lceil n)\right.$ are stable. Clearly, for any $\eta \in U^{T(X)}$, 
$\left\langle\eta, \tau, r_{m}\right\rangle \in L$ for some $\tau \prec T(X)$ and $m$. For any $\eta \in U^{T(X)}$ where $|\eta|>t_{X \nmid n}$ and $\left\langle\eta, \tau, r_{m}\right\rangle$ enters $L$ after stage $s$, we must have $m \geq n$.

By the fact that $L$ is a bounded request set, we have some constant $c$ such that for almost every $\sigma, K(\sigma) \leq r_{m} K^{T(X)}(\sigma)+c$ for some $m \geq n$, and so

$$
K(\sigma) \leq r_{n} K^{T(X)}(\sigma)+c .
$$

That is, we have for almost every $\sigma$,

$$
\frac{K^{T(X)}(\sigma)}{K(\sigma)} \geq \frac{n+1}{n+2}-\frac{c}{K(\sigma) r_{n}}
$$

Hence $T(X)$ is lowish for $K$.

This completes the proof of Theorem 5.1.

\section{Relationship to other DEGReE Classes}

In this section we make a few observations about the degrees of sequences that are low for dimension. The first is that if $A \in 2^{\omega}$ is low for dimension, then it is jump-traceable with a reasonably slow-growing bound. We recall the necessary definitions. A function $h: \omega \rightarrow \omega$ is an order function if it is computable, nondecreasing and unbounded. If $h$ is an order function, then $A \in 2^{\omega}$ is jump-traceable with bound $h$ if for every partial $A$-computable function $f$, there is a c.e. "trace" (sequence of c.e. sets) $\left\{T_{e}\right\}_{e \in \omega}$ such that

$$
(\forall e)\left[\left|T_{e}\right| \leq h(e) \text { and if } f(e) \downarrow \text {, then } f(e) \in T_{e}\right] .
$$

We could relax the above statement to only hold for sufficiently large $e$, and the class of reals so defined would remain unchanged. We say that $A$ is jump-traceable if $A$ is jump-traceable with some bound.

Theorem 6.1. Suppose that $A \in 2^{\omega}$ is low for dimension. Then for every $\varepsilon>0$, $A$ is jump-traceable with bound $n^{\varepsilon}$.

This is an adaptation of a proof found in Nies [18] for the jump-traceability of $K$-trivials.

Proof. Let $A \in 2^{\omega}$ be low for dimension, hence lowish for $K$. Fix $f$ partial $A$ computable. Then for sufficiently long $\sigma, K(\sigma)<(1+\varepsilon / 4) K^{A}(\sigma)$. In particular, $K(n, f(n))<(1+\varepsilon / 4) K^{A}(n, f(n))$ for sufficiently large $n$ in the domain of $f$, where $K(n, m)$ is the prefix-free complexity of the pair $\langle n, m\rangle$. Since $K^{A}(n, f(n))<^{+}$ $K^{A}(n)<^{+} K(n)$, we have $K(n, f(n))<(1+\varepsilon / 3) K(n)$ for sufficiently large $n$ in the domain of $f$.

Fix a universal prefix-free machine $U$. We enumerate a c.e. set $W$ by enumerating $\langle n, d\rangle$ into $W$ when we see $\sum_{c} \sum_{U(\sigma)=\langle n, c\rangle} 2^{-|\sigma|}>2^{-d}$. For each $n$, let $d_{n}$ be least such that we enumerate $\left\langle n, d_{n}\right\rangle$ into $W$.

Then

$$
\sum_{\langle n, d\rangle \in W} 2^{-d} \leq \sum_{n} \sum_{d \geq d_{n}} 2^{-d}=2 \sum_{n} 2^{-d_{n}} \leq 2 \sum_{n, c} \sum_{U(\sigma)=\langle n, c\rangle} 2^{-|\sigma|}<2
$$

and so $W$ is a bounded request set.

Hence there is a $b$ such that $K(n) \leq d_{n}+b$. Since $\langle n, K(n)-b-1\rangle$ was not enumerated into $W$, it follows that $\sum_{U(\sigma)=\langle n, c\rangle} 2^{-|\sigma|} \leq 2^{b+1-K(n)}$. So if we count the number of distinct $c$ with $K(n, c) \leq(1+\varepsilon / 3) K(n)$, since each contributes at 
least $2^{-(1+\varepsilon / 3) K(n)}$ to this sum, there can be at most $2^{b+1+(\varepsilon / 3) K(n)}$ such $c$. Since $K(n) \leq 2 \log n$, we see that there are at most $B n^{2 \varepsilon / 3}$ such $c$ for some constant $B$, and if $n$ is in the domain of $f$, then $f(n)$ is one such $c$.

We now build our trace as follows: We approximate $K(n)$ from above, beginning with $K_{0}(n)=2 \log n$. At stage $s$, if we see a $c$ such that $K_{s}(n, c) \leq(1+\varepsilon / 3) K_{s}(n)$, and we have not yet enumerated $B n^{2 \varepsilon / 3}$ many such $c$ for this current guess of $K(n)$, then we enumerate $c$ into $T_{n}$.

Since $K_{s}(n)$ is non-increasing and positive, it takes on at most $2 \log n$ distinct values. For each such value, we enumerate at most $B n^{2 \varepsilon / 3}$ elements into $T_{n}$, and so $\left|T_{n}\right| \leq B n^{2 \varepsilon / 3} \cdot 2 \log n<n^{\varepsilon}$ for sufficiently large $n$. If $n$ is in the domain of $f$, then once $K_{s}(n)$ takes its final value, $f(n)$ will be one of the $c$ we enumerate into $T_{n}$. Thus $\left\{T_{n}\right\}_{n \in \omega}$ is a trace for $f$ with bound $n^{\varepsilon}$.

Nies [18] constructed a perfect $\Pi_{1}^{0}$-class such that every element is jump-traceable with bound $2 \cdot 4^{e}$. He remarked that his argument could be "massaged a bit to obtain a bound close to $2^{e}$." Together with Theorem 5.1, we get a much better bound:

Corollary 6.2. For any $\varepsilon>0$, there is a perfect $\Pi_{1}^{0}$-class $P \subseteq 2^{\omega}$ such that every $A \in P$ is jump-traceable with bound $n^{\varepsilon}$.

Hence there are continuum many $n^{\varepsilon}$ jump-traceable sequences. ${ }^{5}$ Downey and Greenberg [5] proved that for a sufficiently slow-growing order function $h: \omega \rightarrow \omega$, every sequence that is jump-traceable with bound $h$ is $\Delta_{2}^{0}$ (and even $K$-trivial). But it is not clear where the transition is between having countably many and continuum many jump-traceable sequences (even allowing for the dependence on the numbering of partial computable functions).

Every jump-traceable sequence $A \in 2^{\omega}$ is $\mathrm{GL}_{1}$, i.e., $A^{\prime} \leq_{T} A \oplus \emptyset^{\prime}$ (see Nies [18]), hence this is true if $A$ is low for dimension. We can also draw some degree-theoretic conclusions from the perfect $\Pi_{1}^{0}$-class $P \subseteq 2^{\omega}$ of low for dimension sequences constructed in Theorem 5.1. Let $T: 2^{<\omega} \rightarrow 2^{<\omega}$ be the function tree such that $P=[T]$. Consider the low for dimension path $A=T\left(\emptyset^{\prime \prime}\right)$, Then

$$
\emptyset^{\prime \prime} \leq_{T} A \oplus T \leq_{T} A \oplus \emptyset^{\prime} \leq_{T} T \oplus \emptyset^{\prime \prime} \leq_{T} \emptyset^{\prime \prime},
$$

so $A^{\prime} \equiv_{T} A \oplus \emptyset^{\prime} \equiv_{T} \emptyset^{\prime \prime}$. In other words, $A$ is high. More generally, for any $C \geq_{T} \emptyset^{\prime}$, the low for dimension sequence $A=T(C)$ satisfies $A^{\prime} \equiv_{T} A \oplus \emptyset^{\prime} \equiv_{T} C$. Finally, it is easy to see that any perfect $\Pi_{1}^{0}$-class must contain a sequence $A \leq_{T} \emptyset^{\prime}$ that is not $\omega$-c.e.

\section{OpEn QUESTIONS}

We highlight three questions related to our work. Two ask about the relationship between lowness for dimension and other notions: lowness for packing dimension and having finite self-information. We also ask to what extent our various characterizations remain equivalent when we pass to partially relativized "two oracle" versions.

\footnotetext{
${ }^{5}$ Independently, Yu and Hirschfeldt showed that for any $\varepsilon>0$, there are continuum many $n^{1+\varepsilon}$ jump-traceable sequences.
} 
Lowness for packing dimension. The effective packing dimension of $X \in 2^{\omega}$ is

$$
\operatorname{Dim}(X)=\limsup _{n \in \omega} \frac{K(X\lceil n)}{n} .
$$

Though it is not clear from this definition, Athreya, Hitchcock, Lutz and Mayordomo [1] proved that effective packing dimension is the effective analogue of packing dimension in the same way that effective dimension is the analogue of Hausdorff dimension. We relativize effective packing definition in the obvious way and consider the corresponding lowness notion:

Definition 7.1. We call $A \in 2^{\omega}$ low for packing dimension if $(\forall X)\left[\operatorname{Dim}^{A}(X) \geq\right.$ $\operatorname{Dim}(X)]$.

How does this notion relate to lowness for (Hausdorff) dimension? Are they equivalent? One direction follows easily from the main theorem:

Corollary 7.2. Lowness for dimension implies lowness for packing dimension.

Proof. Assume that $A \in 2^{\omega}$ is low for dimension, hence lowish for $K$. Then for any $X \in 2^{\omega}$,

$$
\begin{aligned}
\operatorname{Dim}^{A}(X)=\limsup _{n \rightarrow \infty} \frac{K^{A}(X\lceil n)}{n} & =\limsup _{n \rightarrow \infty} \frac{K(X\lceil n)}{n} \frac{K^{A}(X\lceil n)}{K(X\lceil n)} \\
& \geq \limsup _{n \rightarrow \infty} \frac{K(X\lceil n)}{n}=\operatorname{Dim}(X) .
\end{aligned}
$$

So $A$ is low for packing dimension.

We expect that lowness for packing dimension is a weaker property than lowness for dimension, but this remains open.

Question 7.3. Does lowness for packing dimension imply lowness for dimension?

Finite self-information. Levin [14] defined the mutual information of $A, B \in 2^{\omega}$ to be

$$
I(A: B)=\log \sum_{\sigma, \tau \in 2<\omega} 2^{-K(\sigma, \tau)-K^{A}(\sigma)-K^{B}(\tau)+K(\sigma)+K(\tau)} .
$$

This is one of several definitions that Levin has put forward to capture the notion of mutual information. Hirschfeldt and Weber [10] investigated the sequences $A \in 2^{\omega}$ that have finite self-information for this definition, i.e., $I(A: A)<\infty$. It is easy to see that if $A \in 2^{\omega}$ is low for randomness, or more specifically, low for $K$, then it has finite self-information. Hirschfeldt and Weber constructed an $A \in 2^{\omega}$ that has finite self-information but is not low for randomness. Not surprisingly, the key to their construction is to ensure that $K^{A}(\sigma)-K(\sigma)$ grows very slowly. They observe that the sequence $A$ they build is both low for (Hausdorff) dimension and low for packing dimension. This led them to ask:

Question 7.4 (Hirschfeldt and Weber [10, Question 20]). What is the relationship between having finite self-information and being low for dimension?

It is possible that neither property implies the other. 
Partial relativization. All of the properties in the main theorem have natural "two oracle" versions. For example, Given $A, B \in 2^{\omega}$, we could consider:

(1) If $X \in 2^{\omega}$ is Martin-Löf random relative to $B$, then $\operatorname{dim}^{A}(X)=1$.

(3) $\left(\forall X \in 2^{\omega}\right)\left[\operatorname{dim}^{A}(X) \geq \operatorname{dim}^{B}(X)\right]$.

(4) If $W \subseteq 2^{<\omega}$ is $A$-c.e., $0 \leq s<t \leq 1$, and $\operatorname{wt}_{s}(W)<1$, then there is a $B$-c.e. set $V$ with $\mathrm{wt}_{t}(V)<1$ such that $[W] \subseteq[V]$.

(7) $\liminf _{\sigma \rightarrow \infty} \frac{K^{A}(\sigma)}{K^{B}(\sigma)} \geq 1$.

If $A \geq_{T} B$, then these and the analogues of the other properties can be shown to be equivalent by relativizing the proofs we have given. What about the general case? Consider Proposition 2.4, where we show that (4) implies (5). The proof is dynamic and requires that the $A$-computable enumeration of $U$ can respond to the enumeration of elements into $Q$. If $Q$ is only assumed to be $B$-c.e., so not necessarily enumerable from $A$, then it is not clear how to modify the proof to make it work. A similar problem arises in Proposition 3.3, where we prove that (6) implies (7).

Question 7.5. Are the two oracle versions of the properties in the main theorem equivalent?

Consider the two oracle versions of properties (b)-(d) in Theorem 1.2. Nies [17] defined $A \leq_{L R} B$ to mean that every sequence that is Martin-Löf random relative to $B$ is Martin-Löf random relative to $A$. Similarly, he defined $A \leq_{L K} B$ to mean that $K^{B}(\sigma) \leq^{+} K^{A}(\sigma)$. These are clearly the two oracle versions of (b) and (c) from the theorem. Kjos-Hanssen [11] proved that $A \leq_{L R} B$ is equivalent to the two oracle version of $(\mathrm{d})$ : every $\Sigma_{1}^{0}[A]$-class of measure less than one is contained in a $\Sigma_{1}^{0}[B]$-class of measure less than one. Kjos-Hanssen, Miller and Solomon [12] built on this result to prove that $A \leq_{L R} B$ if and only if $A \leq_{L K} B$. So the analogy with lowness for randomness might suggest a positive answer to Question 7.5. However, not all results relativize in this way. Write $A \leq_{W 2 R} B$ to mean that every sequence that is weak 2-random relative to $B$ is weak 2-random relative to $A$. Lowness for randomness is equivalent to lowness for weak 2-randomness (by Kjos-Hanssen, Miller and Solomon [12] and Nies [19]), but $A \leq_{L R} B$ is not equivalent to $A \leq_{W 2 R} B$ (by Barmpalias, Miller and Nies [2]).

In partial answer to the question, we can show that the two oracle versions of lowness for dimension and the $\Sigma_{1}^{0}$-covering property are equivalent.

Proposition 7.6. In the two oracle case, (3) $\Leftrightarrow(4)$.

Proof. $\Rightarrow$ : Note that the proof of $(3) \Rightarrow(4)$ in Proposition 2.3 works for the two oracle versions.

$\Leftarrow$ : Assume that $A, B \in 2^{\omega}$ satisfy the two oracle version of property (4). Fix $X \in 2^{\omega}$ and let $s$ and $t$ be rational numbers such that $\operatorname{dim}^{A}(X)<s<t$. Let $W=\left\{\sigma \in 2^{<\omega}: K^{A}(\sigma) \leq s|\sigma|\right\}$. So $W$ is an $A$-c.e. set, $\operatorname{wt}_{s}(W)<1$, and every tail of $X$ is in $[W]$. By assumption, there is a $B$-c.e. set $V$ such that $w_{t}(V)<1$ and $[V] \supseteq[W]$. We have $X \in\left[W^{n}\right] \subseteq\left[V^{n}\right]$ for every $n \in \omega$, so $\operatorname{dim}^{B}(X) \leq t$ by Lemma 2.2 relativized to $B$. But $t$ was any rational greater than $\operatorname{dim}^{A}(X)$, so $\operatorname{dim}^{B}(X) \leq \operatorname{dim}^{A}(X)$. 


\section{REFERENCES}

[1] Krishna B. Athreya, John M. Hitchcock, Jack H. Lutz, and Elvira Mayordomo. Effective strong dimension in algorithmic information and computational complexity. In STACS 2004, volume 2996 of Lecture Notes in Comput. Sci., pages 632-643. Springer, Berlin, 2004.

[2] George Barmpalias, Joseph S. Miller, and André Nies. Randomness notions and partial relativization. Israel J. Math., 191(2):791-816, 2012.

[3] Gregory J. Chaitin. Information-theoretic characterizations of recursive infinite strings. Theoret. Comput. Sci., 2(1):45-48, 1976.

[4] Gregory J. Chaitin. Algorithmic information theory. IBM J. Res. Develop., 21(4):350-359, 1977.

[5] Rodney G. Downey and Noam Greenberg. Strong jump-traceability II: K-triviality. Israel J. Math., 191(2):647-665, 2012.

[6] Rodney G. Downey and Denis R. Hirschfeldt. Algorithmic randomness and complexity. Theory and Applications of Computability. Springer, New York, 2010.

[7] Rodney G. Downey, Denis R. Hirschfeldt, André Nies, and Frank Stephan. Trivial reals. In Proceedings of the 7th and 8th Asian Logic Conferences, pages 103-131, Singapore, 2003. Singapore Univ. Press.

[8] Péter Gács. Every sequence is reducible to a random one. Inform. and Control, 70(2-3):186$192,1986$.

[9] Ian Herbert. A perfect set of reals with finite self-information. J. Symbolic Logic, 78(4):12291246, 2013.

[10] Denis R. Hirschfeldt and Rebecca Weber. Finite self-information. Computability, 1(1):85-98, 2012.

[11] Bjørn Kjos-Hanssen. Low for random reals and positive-measure domination. Proc. Amer. Math. Soc., 135(11):3703-3709, 2007.

[12] Bjørn Kjos-Hanssen, Joseph S. Miller, and Reed Solomon. Lowness notions, measure and domination. J. Lond. Math. Soc. (2), 85(3):869-888, 2012.

[13] Antonín Kučera. Measure, $\Pi_{1}^{0}$-classes and complete extensions of PA. In Recursion theory week (Oberwolfach, 1984), volume 1141 of Lecture Notes in Math., pages 245-259. Springer, Berlin, 1985.

[14] Leonid A. Levin. Laws on the conservation (zero increase) of information, and questions on the foundations of probability theory. Problemy Peredači Informacii, 10(3):30-35, 1974.

[15] Jack H. Lutz. Gales and the constructive dimension of individual sequences. In Automata, languages and programming (Geneva, 2000), volume 1853 of Lecture Notes in Comput. Sci., pages 902-913. Springer, Berlin, 2000.

[16] Elvira Mayordomo. A Kolmogorov complexity characterization of constructive Hausdorff dimension. Inform. Process. Lett., 84(1):1-3, 2002.

[17] André Nies. Lowness properties and randomness. Adv. Math., 197(1):274-305, 2005.

[18] André Nies. Reals which compute little. In Logic Colloquium '02, volume 27 of Lect. Notes Log., pages 261-275. Assoc. Symbol. Logic, La Jolla, CA, 2006.

[19] André Nies. Computability and randomness, volume 51 of Oxford Logic Guides. Oxford University Press, Oxford, 2009.

[20] Boris Ya. Ryabko. Coding of combinatorial sources and Hausdorff dimension. Dokl. Akad. Nauk SSSR, 277(5):1066-1070, 1984.

[21] Stephen G. Simpson. Almost everywhere domination and superhighness. MLQ Math. Log. Q., 53(4-5):462-482, 2007. 
(Lempp, Miller) Department of Mathematics, University of Wisconsin, Madison, Wi 53706-1388, USA

E-mail address: lempp@math.wisc.edu

E-mail address: jmiller@math.wisc.edu

(Ng) Division of Mathematical Sciences, School of Physical \& Mathematical Sciences, College of Science, Nanyang Technological University, Singapore

E-mail address: kmng@ntu.edu.sg

(Turetsky) Kurt Gödel Research Center, University of Vienna, 1090 Vienna, Austria E-mail address: turetsd4@univie.ac.at

(Weber) White River Junction, VT 05001, USA

E-mail address: rweber@alumni.nd.edu 